I.L. Tan, MBBS, FRACP

J.C. McArthur, MBBS, $\mathrm{MPH}$

D.B. Clifford, MD

E.O. Major, $\mathrm{PhD}$

A. Nath, MD

Address correspondence and reprint requests to Dr. Avindra Nath, Building 10/7C-103, 10 Center Drive, NIH, Bethesda, MD 20892

natha@ninds.nih.gov

\title{
Immune reconstitution inflammatory syndrome in natalizumab-associated PML
}

\section{ABSTRACT}

Objective: To study the outcome of patients with multiple sclerosis (MS) and with natalizumabassociated progressive multifocal leukoencephalopathy (PML) and immune reconstitution inflammatory syndrome (IRIS).

Methods: MedWatch reports from Biogen-Idec (manufacturer of natalizumab, Tysabri ${ }^{\circledR}$ ) were reviewed which comprised all 42 cases of natalizumab-related PML cases since its reintroduction until March 2010.

Results: All except 2 patients with natalizumab-related PML were managed by discontinuation of natalizumab and plasmapheresis/immunoadsorption (PLEX/IA). Seventeen patients had contrast enhancement of PML lesions on neuroimaging at the time of diagnosis before withdrawal/removal of natalizumab (early-PML-IRIS) and 23 patients developed contrast enhancement only after withdrawal/removal of natalizumab (late-PML-IRIS). All patients developed IRIS. IRIS was defined as worsening of neurologic deficits during the immune reconstitution following discontinuation of natalizumab, corroborated by inflammatory changes on neuroimaging. Following PLEX/IA, JC viral load in CSF increased by $>10$ fold in those with early-PML-IRIS but $<2$ fold in late-PML-IRIS. IRIS developed earlier and was more severe in early-PML-IRIS $(p<0.05)$. At the last follow-up, all patients had worse EDSS scores but this was higher in patients with early-PML-IRIS compared to those with late-PML-IRIS ( $p>0.05$ ). Mortality was comparable between the 2 groups, $29.4 \pm$ $11 \%$ vs $21.7 \pm 8.8 \%$. Corticosteroid therapy during IRIS was associated with better Expanded Disability Status Scale outcome, $p<0.05$.

Conclusion: Early immunologic rebound in natalizumab-associated PML has worse survival and neurologic outcome. PLEX/IA may accelerate IRIS and its impact on the final outcome is unclear. Corticosteroid therapy provides a modest benefit and needs to be systemically studied in a controlled manner in the management of natalizumab-associated PML-IRIS. Neurology ${ }^{\circledR 2011 ; 77: 1061-1067}$

\section{GLOSSARY}

$\mathbf{C I}=$ confidence interval; EDSS = Expanded Disability Status Scale; IA = immunoadsorption; IRIS = immune reconstitution inflammatory syndrome; $\mathbf{M S}=$ multiple sclerosis; PLEX = plasmapheresis; PML = progressive multifocal leukoencephalopathy .

Progressive multifocal leukoencephalopathy (PML) is an uncommon demyelinating disorder of the CNS that occurs in immunocompromised individuals, especially those with HIV infection. ${ }^{1}$ PML may occur as a result of reactivation of latent JCV in peripheral reservoirs that then invades the CNS. ${ }^{2}$ Recently, biological therapies for autoimmune conditions have resulted in increasing incidence rates of this often life-threatening complication. ${ }^{3-5}$ Some of the monoclonal antibody therapies do not directly suppress immunity but rather dramatically alter normal immune functions or surveillance. ${ }^{6}$

Natalizumab (Tysabri ${ }^{\circledR}$, Biogen-Idec Inc, Cambridge, MA) is a humanized monoclonal antibody directed against the cellular adhesion molecule $\alpha 4$-integrin and is used in the treatment of multiple sclerosis (MS). By inhibiting the egress of lymphocytes from the blood vessels, it markedly reduces inflammation in the CNS.7,8

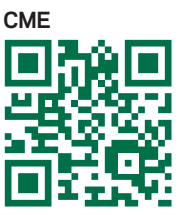

From the Department of Neurology (I.L.T., J.C.M., A.N.), Johns Hopkins School of Medicine, Baltimore, MD; Department of Neurology (D.B.C.), Washington University, St Louis, MO; and Laboratory of Molecular Medicine and Neuroscience (E.O.M.), NINDS, NIH, Bethesda, MD. Study funding: Supported in part by NIH grants: 1P30MH075673, UL1RR025005, R01NS056884.

Disclosure: Author disclosures are provided at the end of the article. 
Three cases of PML associated with natalizumab treatment were first reported in 2005 after which natalizumab was temporarily taken off the market. ${ }^{9-11}$ Between its reintroduction in November 2006 and March 2010, 42 postmarketing cases of confirmed PML had been reported in patients with MS treated with natalizumab.

Plasma exchange (PLEX) or immunoadsorption (IA) has been used to remove natalizumab, thus restoring lymphocyte trafficking into the brain in patients who developed PML. ${ }^{12}$ Paradoxically, the effective removal of natalizumab and sudden restoration of cellular immunity may cause worsening of neurologic deficits, consistent with the development of immune reconstitution inflammatory syndrome (IRIS).

In this retrospective study, we have characterized the clinical manifestations and prognostic factors of natalizumab-associated PML and the timing of IRIS in this setting.

METHODS We analyzed data from MedWatch reports from November 2006 to March 2010 obtained from Biogen-Idec, the manufacturer of natalizumab. During this period, a total of 42 cases of confirmed PML had been reported globally. Follow-up reports were obtained on these individuals until August 2010. We excluded 2 patients from the 42 cases because they had not received PLEX/IA. We reviewed information on clinical features, virologic and immunologic analyses, neuroimaging, treatment, and outcome. Our outcome analysis included patients with available data with regards to their Expanded Disability Status Scale (EDSS) score at various time points (table).

PML-IRIS in this cohort was defined by the following clinical criteria: 1) individuals treated with natalizumab for MS, 2) the diagnosis of PML was established by detection of JC virus DNA in the CSF or by immunohistochemistry on brain tissue following biopsy, 3) worsening neurologic symptoms and signs following cessation of natalizumab or removal of natalizumab by PLEX/IA, and 4) evidence of extension of lesions with contrast enhancement or mass effect on neuroimaging in lesions considered to be due to PML.

The neuroimaging in this cohort of patients was atypical of classic PML, demonstrating inflammatory features of contrast enhancement and mass effect, like those observed in HIVassociated PML-IRIS. ${ }^{13}$

We grouped the patients into early-PML-IRIS if there was contrast enhancement on their neuroimaging in the PML lesions at the time of diagnosis of PML and before the withdrawal/ removal of natalizumab and late-PML-IRIS if contrast enhancement or worsening features of PML lesions occurred only after withdrawal/removal of natalizumab. We compared the clinical characteristics and outcomes between the 2 groups.

Standard protocol approvals, registrations, and patient consents. Since this was an analysis of a deidentified MedWatch database, a formal review by the Institutional Review Board at our institution was not required.

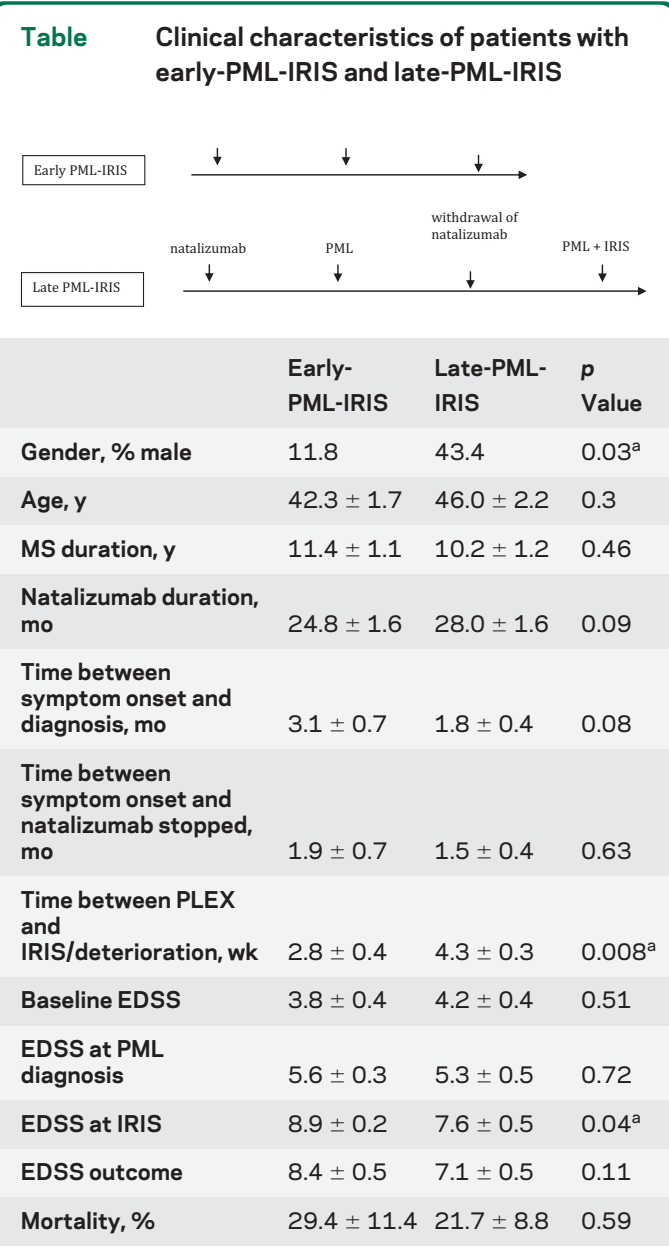

Abbreviations: EDSS = Expanded Disability Status Scale; IRIS = immune reconstitution inflammatory syndrome; $\mathrm{MS}=$ multiple sclerosis; PLEX = plasmapheresis; PML = progressive multifocal leukoencephalopathy.

a Significant.

Statistical analysis. Multivariate regression was used to predict correlations. Differences between the 2 groups were assessed using $t$ test. All statistical analyses were performed with Graph Pad Prism software version 5.0a (GraphPad Software, San Diego, CA). All values are presented as mean \pm SEM.

RESULTS Characteristics of patients. There were 12 men and 28 women, with a mean age of $44.5 \pm 1.5$ years (range 27-67 years). The mean duration of MS was $10.7 \pm 0.8$ years (range 15 months-20 years). The mean duration of treatment with natalizumab was $26.6 \pm 1.2$ months (range 12-41 months). All but one patient had been previously exposed to disease-modifying agents including $\beta$-interferon or glatiramer. Nineteen patients had previously been treated with some form of immunosuppressive therapy including azathioprine, mycophenolate mofetil, methotrexate, cyclophosphamide, or mitoxantrone. Neither previous exposure to immunosuppressive agents ( $\beta=0.04,95 \%$ confidence interval $[\mathrm{CI}]=-0.12$ to 0.035$)$ nor duration of immunosuppressives $(\beta=-2.3,95 \% \mathrm{CI}=-5.4$ to 
0.84) significantly impacted the outcome. Previous exposure to immunosuppressive agents was documented in $29.4 \%$ in early-PML-IRIS compared to $56.5 \%$ in the late-PML-IRIS, $p>0.05$. One patient had previously received stem cell infusion and another patient had autologous bone marrow transplantation. The duration of natalizumab therapy also did not impact the outcome.

In all patients in this series, the diagnosis of PML was established by the demonstration of JC virus in the CSF or brain tissue. The presenting symptoms of PML included a combination of cognitive and behavioral changes $(54.7 \%)$, motor weakness (45.2\%), sensory complaints $(7.14 \%)$, speech disturbance (23.8\%), visual impairment (40.5\%), and seizures (14.2\%). The mean baseline EDSS while stable on natalizumab therapy was $4.1 \pm 0.3$ (range $1-8$ ) and the EDSS at the time of PML diagnosis had increased to $5.4 \pm 0.3$ (range $2-9$ ). There were more men in the late-PML-IRIS group $(11.8 \%$ in earlyPML-IRIS vs $43.4 \%$ in late-PML-IRIS, $p<0.05$ ). While this may be a statistical anomaly as the number of individuals in each group was small, it may also suggest that there may be a more robust immune response to JCV in females, or that the immune cells were able to cross the blood-brain barrier despite natalizumab in females, so that there was a higher ratio of females in the early-PML-IRIS group. There was no significant difference in other clinical characteristics between the 2 groups (table).

The mean delay between symptom onset and diagnosis of PML was $2.3 \pm 0.4$ months (range 1 week-10 months) with no significant difference between the 2 groups. The delay in diagnosis could be attributed to 1) the presenting symptoms as well as neuroimaging of PML being misinterpreted as relapses of MS ( $\mathrm{n}=15)$; 2) the high proportion of patients presenting initially with cognitive changes, which was more subtle and subjective than physical disability ( $\mathrm{n}=9)$; and 3 ) the lack of sensitivity of some local laboratories in detecting JCV in CSF, resulting in initially false-negative results $(n=5)$. In these cases, CSF samples concurrently analyzed in Dr. Major's laboratory were positive for JCV. There was no significant difference in the interval between symptom onset and natalizumab cessation between the 2 groups (table).

Relationship of IRIS to PLEX/IA. All but 2 patients received PLEX/IA following the diagnosis of PML. Most patients had 3-5 sessions of PLEX/IA, administered on alternate days with $1-1.5 \times$ plasma volumes in each exchange. Patients who received only 3 PLEX $(n=6)$ either could not tolerate PLEX or worsened while on PLEX. All patients initially improved or stabilized with the withdrawal of natali- zumab and plasmapheresis/IA, but paradoxically worsened subsequently. The duration from PLEX/IA to subsequent deterioration was shorter in earlyPML-IRIS compared to late-PML-IRIS. The time interval between PLEX/IA and the worsening of IRIS was $2.8 \pm 0.4$ weeks in early-PML-IRIS and $4.3 \pm 0.3$ weeks for development of IRIS in latePML-IRIS $(p<0.05)$. EDSS at the time of development of IRIS was $8.9 \pm 0.2$ in early-PML-IRIS and $7.6 \pm 0.5$ in late-PML-IRIS $(p<0.05)$. The EDSS outcome at the last follow-up of $8.4 \pm 0.9$ months (range 1-24 months) was $8.4 \pm 0.5$ in early-PMLIRIS and $7.1 \pm 0.5$ in late-PML-IRIS $(p>0.05)$. There was no significant difference in the outcome of patients who received 3 ( $\mathrm{n}=6$, EDSS outcome $=$ $8.8 \pm 0.4)$ vs 5 sessions of PLEX/IA $(n=24$, EDSS outcome $=8.0 \pm 0.4, p=\mathrm{NS})$.

Two patients did not receive PLEX/IA. One patient was diagnosed with PML-IRIS by brain biopsy 5 months after the cessation of natalizumab. The biopsy showed marked inflammation with plasma cells in the white matter. The astrocytes were markedly enlarged, some with atypical nuclei. The presence of JCV was confirmed by immunohistochemistry. This patient had developed symptoms of IRIS 3 months post natalizumab cessation, with mild worsening of ataxia accompanied by an expanding right frontal juxtacortical lesion and contrast enhancement on neuroimaging. The patient received no PML-specific treatment. At the last follow-up (6 months post brain biopsy), the patient was ambulating with a cane. The EDSS had remained unchanged since the time of the diagnosis of PML. Neither did the second patient receive any PML-specific therapy. The patient deteriorated about 2.5 months after natalizumab was discontinued, with EDSS of 8.

One patient had further cycles of PLEX upon development of symptoms of IRIS. This patient had received 33 doses of natalizumab when he developed progressive right hemiparesis. MRI of the brain showed T2/fluid-attenuated inversion recovery signal hyperintensity lesions with patchy enhancement in both hemispheres, worse on the left. He was initially treated as a relapse of MS. He received PLEX when JCV DNA was detected in the CSF. There was some initial improvement with PLEX, but he deteriorated with evidence of IRIS about 4 weeks after PLEX. He was retreated with PLEX and corticosteroids, but died 3 weeks later.

There was no correlation between the JCV load at the time of diagnosis of PML and subsequent outcome. Nine pairs of CSF samples were available, one around the time of initial PML diagnosis and the second around the time of worsening neurologic deficits contemporaneous with the development of 
Figure 1 Change of CSF JC viral load before plasma exchange (PLEX) and postPLEX (around the time of immune reconstitution inflammatory syndrome)

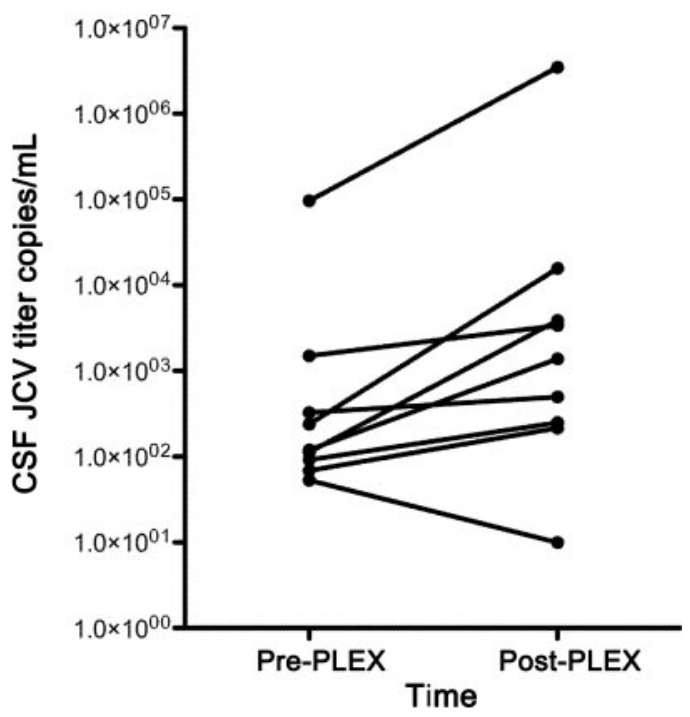

IRIS. All but one patient had an increase of CSF JCV load at the time of development IRIS. In earlyPML-IRIS, there was an increased JCV titer of $24.9 \pm 9.7$-fold at the time of IRIS. In contrast, in late-PML-IRIS, the increase in JCV titer was only $1.1 \pm 0.3$-fold, $p=$ NS (figure 1). Only 4 patients had multiple CSF samples (figure 2). There was an increase in CSF JCV titer following PLEX, peaking around day 50 after the initiation of PLEX. JCV load in CSF improved and often became undetectable over time. Corticosteroid administration coincided with either stabilization or a drop in JCV load.

Effect of treatment of PML and IRIS. In addition to PLEX/IA, mefloquine and mirtazapine were prescribed for some patients at the time of the diagnosis of PML. Twenty-three patients received mefloquine at the time of diagnosis of PML (9 early-PML-IRIS, 14 latePML-IRIS), and in one patient it was started when late IRIS developed. Twenty-two patients received mirtazapine (9 early-PML-IRIS, 13 late-PML-IRIS). Sixteen patients received both mefloquine and mirtazapine (5 patients early-PML-IRIS, 11 patients late-PML-IRIS). There was no difference between the 2 groups in the use of either mefloquine or mirtazapine use. Further subanalysis showed that in the late-PML-IRIS group, the EDSS of those who received mefloquine was $8.9 \pm 0.4(\mathrm{n}=10)$ compared to $8.9 \pm 0.6(\mathrm{n}=7)$ in those who did not receive mefloquine $(p=N S)$, while in those with late-PML-IRIS, the mean EDSS in mefloquine recipients was $8.2 \pm 0.7$ compared to $5.7 \pm 0.9$ $(p=\mathrm{NS})$ in those who were not treated with mefloquine. Similarly, there was no significant differ-

Figure 2 CSF JC viral load at various time points in relation to plasma exchange (PLEX) and steroid administration
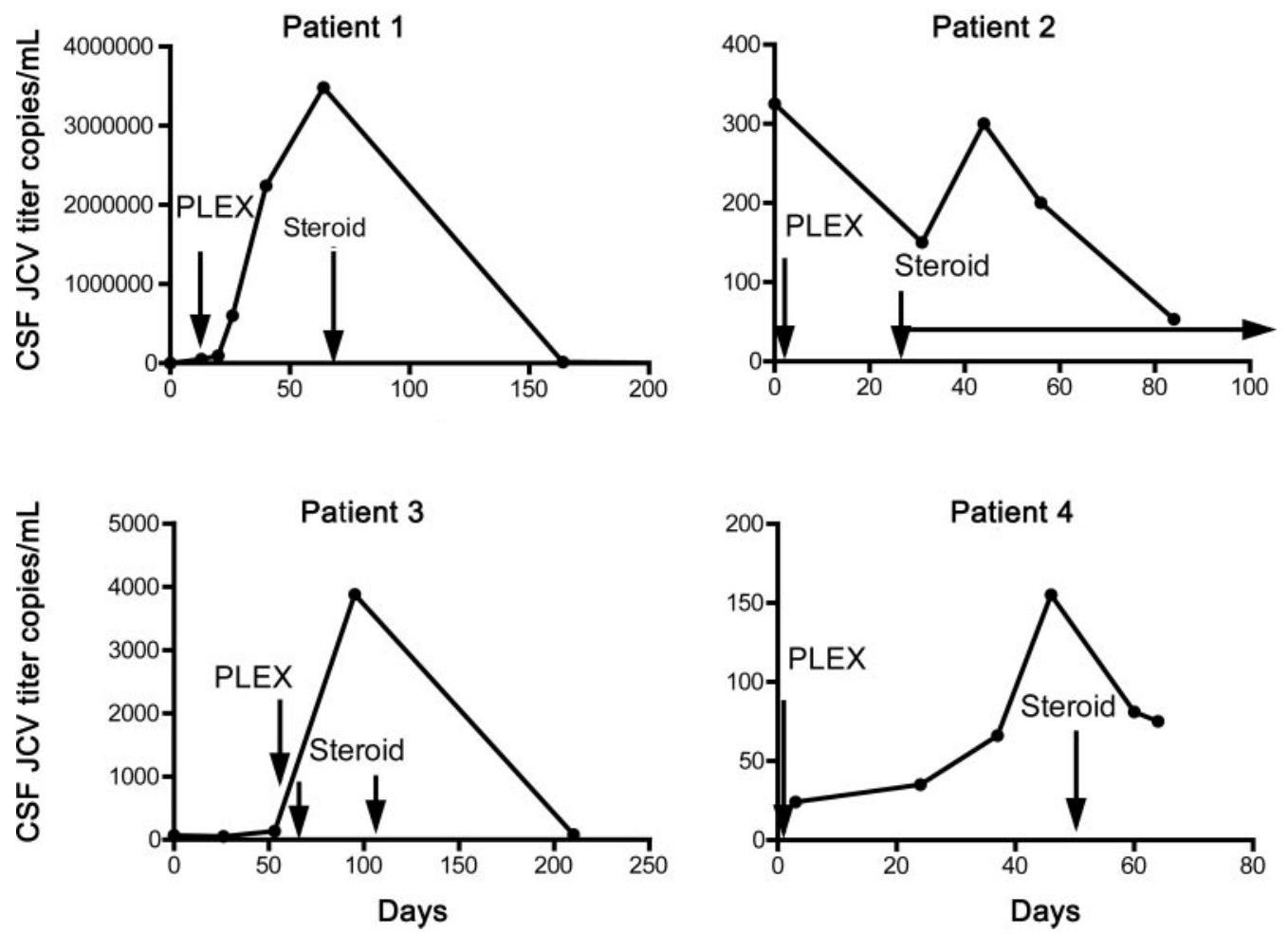
ence in the mirtazapine-treated groups. Two patients received cytarabine and both patients died.

Corticosteroids usage. Some patients were treated with corticosteroids at the initial presentation of PML when their symptoms were mistaken for an MS relapse. The use of corticosteroids was more common in the early-PML-IRIS group, probably influenced by contrast enhancement on the initial neuroimaging. However, the early use of corticosteroids ( $\mathrm{n}=13$ ) had no impact on the eventual outcome in this group; the mean follow-up EDSS was $8.7 \pm 0.4$ in the treated group and $8.8 \pm 0.3$ in the group not treated with early corticosteroid ( $p=\mathrm{NS}$ ). In late-PML-IRIS, the follow-up EDSS of those treated with early corticosteroids was $8.8 \pm 0.8(\mathrm{n}=$ 6) compared to $6.3 \pm 0.7(n=12)$ in those not treated with early corticosteroids $(p=0.05)$.

When IRIS developed, corticosteroids were used in the majority of the patients, some with pulse corticosteroids alone, while others had a more prolonged corticosteroid course with slowly tapering regimens variably over 2-6 weeks. The follow-up EDSS of patients treated with corticosteroids ( $\mathrm{n}=$ 22) was $7.4 \pm 0.5$ compared to $9.1 \pm 0.6$ in the untreated group $(n=9), p<0.05$. The EDSS of patients treated with IV methylprednisolone only $(\mathrm{n}=10)$ was $8.1 \pm 0.5$ vs $6.7 \pm 0.7$ in patients treated with IV methylprednisolone and a minimum of 1 month of oral tapering of corticosteroid ( $\mathrm{n}=$ 12), $p=$ NS.

Outcomes. The overall outcome was worse in those who developed early-PML-IRIS compared to those with late-PML-IRIS. The mean follow-up EDSS was $8.7 \pm 0.3$ in early-PML-IRIS compared to latePML-IRIS $(7.1 \pm 0.6, p>0.05)$. The overall mortality rate was $29 \pm 11 \%$ vs $22 \pm 9 \%(p=N S)$ in early-PML-IRIS compared to late-PML-IRIS.

DISCUSSION Our data suggest that an inflammatory form of PML developed in all cases of natalizumab-associated PML. Patients appeared to be differentiated by the time course and patterns of clinical worsening and contrast enhancement relative to PLEX after the diagnosis of PML into early and late IRIS. Even before cessation of natalizumab, some of the patients had evidence of inflammatory PML based on contrast-enhancing PML lesions, and these had earlier clinical evolution after PLEX/IA and hence we termed this phenomenon early-PMLIRIS. Cessation of natalizumab alone can cause immunologic rebound with IRIS, typically with restoration of immune surveillance in the CNS (about 3 months postcessation of natalizumab as predicted by the prolonged biologic activity of this agent). In our series, the attempted removal of natalizumab with PLEX/IA was followed by the development or worsening of IRIS in about 3 weeks in the early-PMLIRIS group and 4.5 weeks in the late-PML-IRIS group. Early-PML-IRIS was associated with worse outcome, both in terms of follow-up EDSS and mortality, which was notably high.

There was often a considerable delay between symptom onset and the actual diagnosis of PML, although neither the delay in diagnosis, the time from symptom onset to stopping natalizumab, nor the time from stopping natalizumab to PLEX/IA could account for the differences between early-PML-IRIS and late-PML-IRIS.

Treatment of PML requires immune reconstitution, i.e., removal of natalizumab. Plasma exchange is effective in accelerating the clearance of natalizumab. Desaturation of $\alpha 4$-integrin $(<50 \%)$ occurs when plasma concentration of natalizumab is $<1 \mu \mathrm{g} / \mathrm{mL}$, which is achieved 15 days after 3 sessions of PLEX or at 82 days without PLEX. ${ }^{14}$ This correlates with the development of IRIS in our cohort; IRIS developed at a mean of 19 days in early-PML-IRIS or at 2-3 months after stopping natalizumab without PLEX.

Strikingly, all patients with natalizumab-associated PML developed IRIS, reasons for which may be as follows: first, natalizumab primarily impacts the trafficking of immune cells, not their competence, resulting in the development of PML-IRIS in the setting of normal immune competence. The sequestrated lymphocytes readily enter the brain to mount a potent immune response when natalizumab is removed. These activated lymphocytes lead to an inflammatory reaction at the site of the PML lesion. Secondly, the peripheral proinflammatory cytokines and activated cells are increased so that when natalizumab is removed, the rebound restoration of immune response is overwhelming. Natalizumab is associated with peripheral sequestration of proinflammatory cells, cytokine-producing $\mathrm{T}$ cells, which may be the direct effect of natalizumab or a secondary effect from peripheral accumulation. ${ }^{15}$ Activated $\mathrm{T}$ cells have been shown to cause neurotoxicity via the release of granzyme B. ${ }^{16}$

The development of IRIS as we operationally defined it was associated with substantial worsening of functional outcome, with a mean EDSS deterioration of 3.1. Perhaps because the inflammatory response was already well-advanced in the early-PML-IRIS patients, they had worse neurologic disability and higher mortality compared to those with late-PMLIRIS. The development of these 2 forms of IRIS is not influenced by prior exposure to immune suppressive drugs. These observations also suggest that 
the 2 forms of IRIS may be pathophysiologically distinct and patients with early-PML-IRIS are able to mount a much stronger immune response. The earlier manifestation of IRIS in this group may be further facilitated by the already breached blood-brain barrier, as evident by contrast enhancement, that allowed more rapid trafficking of proinflammatory cells into the CNS resulting in marked increase of JCV load in CSF, presumably due to more effective killing of JCV-infected oligodendrocytes leading to release of viral DNA into the CSF. Unfortunately, the overwhelming immune and inflammatory response can be paradoxical and harmful with potentially fatal results. Viral persistence has been reported in some cases, despite PLEX/IA and robust IRIS. ${ }^{17}$ Similarly in HIV-infected patients with PML, 2 forms of IRIS have been recognized. ${ }^{18}$ In some patients PML occurs only following antiretroviral therapy and is associated with an inflammatory reaction and is hence termed simultaneous PML-IRIS. In that sense it bears similarity to early-PML-IRIS. In other HIV-infected patients known to have PML, IRIS develops only after subsequent initiation of antiretroviral therapy. This is termed delayed PML-IRIS and is similar to late PML-IRIS. While there was no difference in the outcome between early and late-PMLIRIS, a previous study in HIV-infected patients suggested that delayed PML-IRIS may have a worse prognosis. ${ }^{13}$

Although the study was not designed to test efficacy, mefloquine and mirtazapine did not appear to affect the outcome of PML in this natalizumab cohort. An ongoing trial of mefloquine (ClinicalTrials.gov identifier: NCT00746941) for PML associated with HIV and other forms of PML was recently concluded and analysis is underway.

An IRIS-like phenomenon has been observed in mycobacterium tuberculosis infections. Adjuvant steroid therapy in the treatment of tuberculous meningitis in HIV-negative patients improves survival. ${ }^{19}$ Corticosteroid treatment in PML-IRIS is anecdotal and treatment trials are lacking.

In our series, corticosteroids were used in various regimens, doses, and formulations, and were administered at various time points during the course of PML and PML-IRIS. Early corticosteroid use may be associated with a worse outcome in the late-PMLIRIS group, although the worse outcome may at least in part be attributed to a delay in PML diagnosis. Early corticosteroids had no effect on the subsequent development of IRIS in the early-PML-IRIS group. Conversely, the administration of corticosteroids following the development of IRIS appeared to be associated with favorable EDSS outcomes. These observations may support the use of corticosteroids for treatment of IRIS and may favor a prolonged course of treatment.

The major limitation of data collection from MedWatch reports was the availability and variability of the clinical information. Most of the reporting physicians remained anonymous and no further data or follow-up information were feasible. The analysis of the clinical information was performed only on available data (table). The use of EDSS, as available from MedWatch, in this cohort was not ideal as this has not been validated to assess severity for PMLIRIS. Nonetheless, as a surrogate, this provided a measure of neurologic function.

IRIS is universal in these patients with MS who developed PML in the setting of natalizumab therapy. IRIS is the major cause of morbidity and mortality in these patients, who as a group had devastating neurologic deficits and a substantial mortality rate. The time to deterioration was shorter in early-PML-IRIS; this warrants prospective studies to investigate if plasmapheresis aggravates or worsens IRIS and if there is a role for less aggressive plasmapheresis. Our findings also suggest that prolonged corticosteroid therapy may be necessary during PML-IRIS associated with natalizumab, but this requires further investigation.

\section{AUTHOR CONTRIBUTIONS}

Dr. Tan: design, analysis, and writing of the manuscript. Dr. McArthur: design, review, and writing of the manuscript. Dr. Clifford: design, review, and writing of the manuscript. Dr. Major: review and writing of the manuscript. Dr. Nath: design, analysis, writing, and review of the manuscript.

\section{DISCLOSURE}

Dr. Tan reports no disclosures. Dr. McArthur serves on a scientific advisory board for CNS Bio Services; receives publishing royalties for Current Therapy in Neurologic Disease, 7th Edition (Mosby, 2006); is an author on patents re: Device for thermal stimulation of small neural fibers and Immunophilin ligand treatment of antiretroviral toxic neuropathy; and receives research support from Biogen Idec, Pfizer Inc., the NIH, the National Multiple Sclerosis Society, and the Foundation for Peripheral Neuropathy. Dr. Clifford serves/has served on scientific advisory boards for Biogen Idec, Elan Corporation, Roche, Forest Laboratories, Inc., Genentech, Inc., GlaxoSmithKline, Millennium Pharmaceuticals, Inc., Schering-Plough Corp., Bristol-Meyers Squibb, and Genzyme Corporation; received speaker honoraria and funding for travel from GlaxoSmithKline, Millennium Pharmaceuticals, Inc., and Genentech Inc.; has received research support from Pfizer Inc, Schering-Plough Corp., Bavarian Nordic, NeurogesX, GlaxoSmithKline, Tibotec Therapeutics, Boehringer Ingelheim, and Gilead Sciences, Inc.; and receives research support from the NIH (NIMH, NINDS, NIAID, and Fogarty Institutes). Dr. Major receives research support from the NIH/NINDS. Dr. Nath serves on scientific advisory boards for Biogen Idec and DioGenix, Inc.; serves as an Associate Editor for the Journal of Neurovirology; may accrue revenue on patents re: Tat as an immunogen, Diosgenin for treatment of neurodegenerative diseases, Role of Kv channels in neuroregeneration and protection, Role of lominoid compounds as neuroprotective agents, and Tat ELISA; has served as a consultant for Nerveda Inc. and Elan Corporation; receives research support from the NIH; and has served as an expert advice in medico-legal cases.

Received January 6, 2011. Accepted in final form April 5, 2011. 


\section{REFERENCES}

1. Koralnik IJ. New insights into progressive multifocal leukoencephalopathy. Curr Opin Neurol 2004;17:365-370.

2. Stüve O, Marra CM, Jerome KR, et al. Immune surveillance in multiple sclerosis patients treated with natalizumab. Ann Neurol 2006;59:743-747.

3. Carson KR, Focosi D, Major EO, et al. Monoclonal antibody-associated progressive multifocal leukoencephalopathy in patients treated with rituximab, natalizumab, and efalizumab: a review from the Research on Adverse Drug Events and Reports (RADAR) Project. Lancet Oncol 2009;10:816-824.

4. Carson KR, Even AM, Richey EA. Progressive multifocal leukoencephalopathy after rituximab therapy in HIVnegative patients: a report of 57 cases from the Research on Adverse Drug Events and Reports Project. Blood 2009; 113:4834-4840.

5. Bellizi A, Barucca V, Fioriti D, et al. Early years of biological agents therapy in Crohn's disease and risk of the human polyomavirus JC reactivation. J Cell Physiol Epub 2010 Apr 16.

6. Major EO. Progressive multifocal leukoencephalopathy in patients on immunomodulatory therapies. Annu Rev Med 2010;61:35-47.

7. Baron JL, Madri JA, Ruddle NH, Hashim G, Janeway CA Jr. Surface expression of alpha- 4 integrin by CD $4 \mathrm{~T}$ cells is required for their entry into brain parenchyma. J Exp Med 1993;177:57-68.

8. Yednock TA, Cannon C, Fritz LC, Sanchez-Madrid F, Steinman L, Karin N. Prevention of experimental autoimmune encephalomyelitis by antibodies against alpha- 4 beta-1 integrin. Nature 1992;356:63-66.

9. Langer-Gould A, Atlas SW, Green AJ, Bollen AW, Pelletier D. Progressive multi- focal leukoencephalopathy in a patient treated with natalizumab. N Engl J Med 2005;353: 375-381.

10. Kleinschmidt-DeMasters BK, Tyler KL. Progressive multifocal leukoencephalopathy complicating treatment with natalizumab and interferon beta-1a for multiple sclerosis. N Engl J Med 2005;353:369-374.

11. Van Assche G, Van Ranst M, Sciot R, et al. Progressive multifocal leukoencephalopathy after natalizumab therapy for Crohn's disease. N Engl J Med 2005;353:362-368.

12. Clifford DB, DeLuca A, Simpson DA, et al. Natalizumab associated progressive multifocal leukoencephalopathy in patients with multiple sclerosis: lessons from 28 cases. Lancet Neurol 2010;9:438-446.

13. Tan K, Roda R, Ostrow L, et al. PML-IRIS in patients with HIV infection: clinical manifestations and treatment with steroids. Neurology 2009;72:1458-1464.

14. Khatri BO, Man S, Giovannoni G, et al. Effect of plasma exchange in accelerating natalizumab clearance and restoring leukocyte function. Neurology 2009;72:402-409.

15. Kivisakk P, Healy BC, Viglietta V, et al. Natalizumab treatment is associated with peripheral sequestration of proinflammatory T cells. Neurology 2009;72:1922-1930.

16. Wang T, Allie R, Conant K, et al. Granzyme B mediates neurotoxicity through a G-protein coupled receptor. FASEB J 2006;20:1209-1211.

17. Ryschkewitsch CF, Jensen PN, Monaco MC, Major EO. JC virus persistence following PML is MS patients treated with natalizumab. Ann Neurol 2010;68:384-391.

18. Berger JR, Levy RM, Flomenhoft D, Dobbs M. Predictive factors for prolonged survival in acquired immunodeficiency syndrome-associated progressive multifocal leukoencephalopathy. Ann Neurol 1998;44:341-349.

19. Thwaites GE, Nguyen DB, Nguyen HD, et al. Dexamethasone for the treatment of tuberculous meningitis in adolescents and adults. N Engl J Med 2004;351:1741-1751.

\section{Don't Just Settle-Learn to Negotiate With Hospitals and Insurers}

Register now for a live AAN webinar, "Win, Lose, or Draw? Negotiating with Hospitals and Insurers" and learn how to effectively negotiate with private insurers and hospital management, build and maintain positive and productive relationships with them, and utilize the AAN's Insurer Relations Toolkit. The 90-minute session led by program director Joel Kaufman, MD, FAAN, takes place at 12:00 p.m., Eastern Time, Tuesday, September 20. The deadline to register is September 16. Physicians earn 1.5 AMA PRA Category 1 Credits $^{\mathrm{TM}}$ per webinar, and non-neurologists (i.e., practice and office managers) will receive a certificate of completion redeemable for credits. You can invite as many attendees to your webinar as you like—you only pay per phone line.

Visit www.aan.com/view/pmb for more information. 